\title{
A Technique for Examining Large Numbers of Bacterial Culture Filtrates by Partition Chromatography
}

\author{
By A. J. WOIWOD \\ The Wellcome Research Laboratories, Beckenham, Kent
}

\begin{abstract}
SUMMARY : A description is given of apparatus and methods which have been used to produce large numbers of satisfactory and reproducible chromatograms of bacterial culture filtrates. Some of the factors which influence the preparation and development of chromatograms are discussed.
\end{abstract}

While studying the nitrogen metabolism of micro-organisms by means of paper chromatography (Woiwod \& Proom, 1948; Proom \& Woiwod, 1949a, b); Linggood \& Woiwod, 1948), it was necessary to prepare large numbers of single-dimensional paper chromatograms. During this work some of the conditions for obtaining reproducible and satisfactory chromatograms were determined. As little detailed descriptive work on the technique of paper chromatography has been published since the original observations of Consden, Gordon \& Martin (1944), description of the apparatus and methods employed in this laboratory is given in the hope that it will prove of use to workers wishing to use this elegant analytical method.

In partition chromatography on paper an organic solvent such as butanol, saturated with water, is allowed to pass over a dried spot of a solution of the material under investigation placed on a strip of filter-paper. The process is carried out in a closed chamber in an atmosphere saturated with water and solvent. The various constituents present in the spot are separated by this process and on completion of the process will have travelled different distances from the starting line. The distance will depend on the distribution coefficient of the substance between water and the organic solvent used and on other physico-chemical factors, some of which are not fully understood. After drying, the paper is examined under ultra-violet light and then sprayed with the appropriate reagent to reveal the positions of the separated constituents of the original spot. In the case of amino-acids and peptides, the reagent is ninhydrin, which produces coloured spots on heating. The ratio of the distance the substance has run to the distance the solvent front has travelled is known as the $R_{\boldsymbol{F}}$ value and varies with the solvent employed. It is a useful means of indicating the approximate position which a substance will occupy on a chromatogram. It cannot be used by itself to identify an amino-acid or peptide with certainty; this can be done only by noting other things in addition, such as shade of colour produced by the ninhydrin, position relative to other aminoacids, $\boldsymbol{R}_{\boldsymbol{F}}$ value in some second solvent and by running on the same chromatogram a control mixture containing known amino-acids. 


\section{APPARATUS AND METHODS}

Tanks and Accessories. Rectangular tanks $2 \mathrm{ft}$. long, $1 \mathrm{ft}$. wide and $3 \mathrm{ft}$. deep, which can be opened at the top, and are capable of accommodating up to eight sheets of chromatograms on filter-paper $22 \frac{1}{2}$ in. by $18 \frac{1}{4}$ in., are used. De-scaled quality stainless steel sheet, $c .0 .06 \mathrm{in}$. thick, is the most satisfactory material.
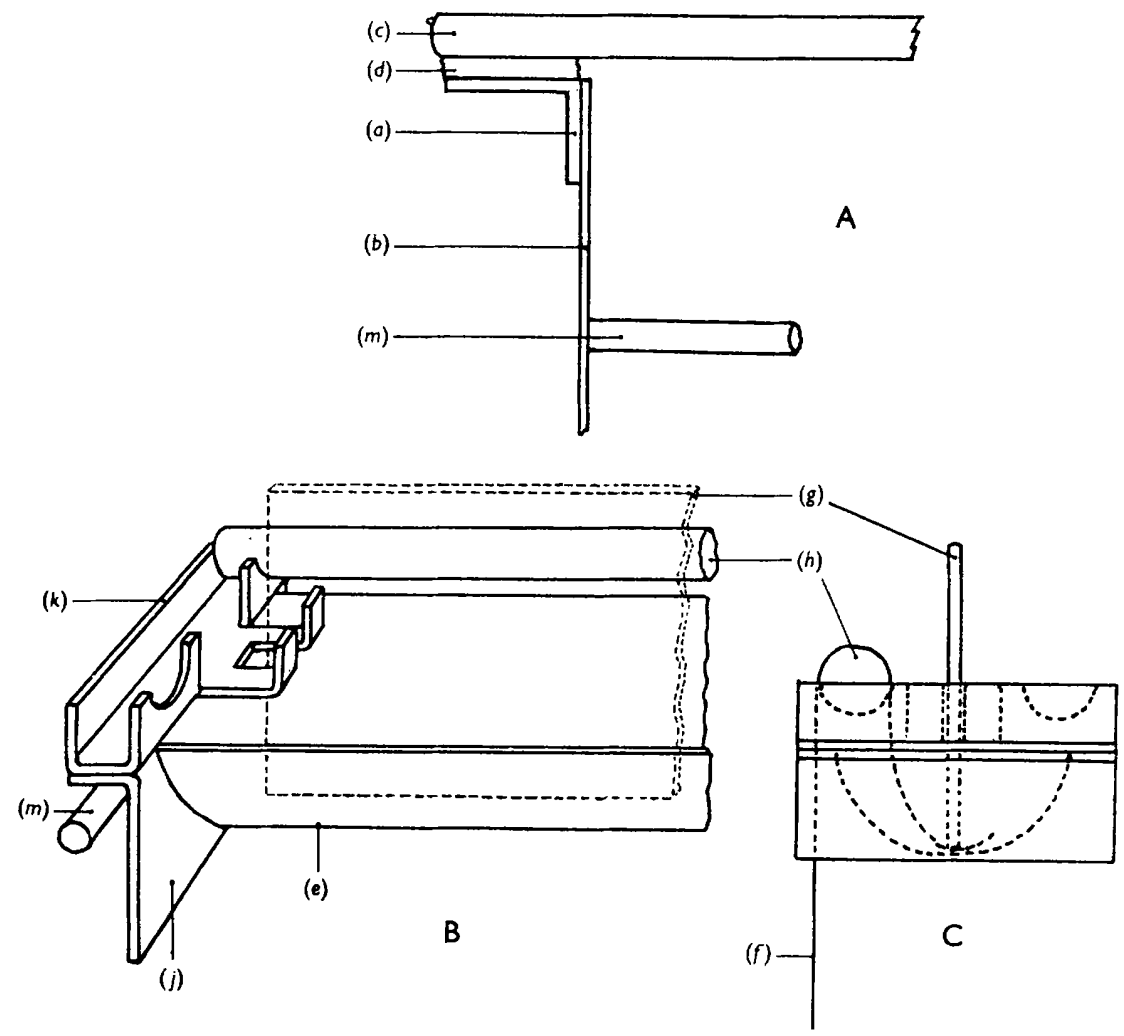

Fig. 1. A, method of sealing tank; B, side view of trough; $C$, end view of trough.

Welded tanks of this material withstand prolonged exposure to $n$-butanol + acetic acid mixture, phenol and 'collidine', with no sign of corrosion. The method of closing the top of the tank to form a solvent-tight seal is shown in Fig. 1A. A welded angle-iron frame (a) is soft solderedyto the top of the tank $(b)$ which is closed by a sheet of plate glass $(c)$ resting on a sponge-rubber gasket $(d)$ fixed to the frame with Bostik $C$ compound'(B.B. Chemical Co. Ltd., Leicester). Removable troughs fitting inside the tank hold the water-saturated solvent. De-scaled stainless steel sheet is satisfactory for the troughs and is more robust than glass. A simple design (Fig. 1 B, C) has enabled easily cleaned troughs of standard size to be produced. The body of the trough $(e), 22 \frac{3}{4} \mathrm{in}$. long, is semicircular in cross-section ( $1 \frac{1}{2} \mathrm{in}$. diam.). One edge of the filter-paper sheet $(f)$ dips into the trough and is held in position by a removable stainless 
steel plate $(g)$. The rest of the paper, after passing over a glass support $(h)$, hangs vertically in the tank. The end-closure $(j)$ and the support $(k)$ which holds the glass rods and stainless steel plate, are welded on to the end of the trough, which is supported in the tank on $\frac{1}{4}$ in. diameter stainless rod $(m)$ fixed $1 \frac{3}{4}$ in. below the top.

Paper and solvents. When single-dimensional chromatograms are being used to study the changes produced by micro-organisms in a complex medium such as an acid-hydrolysate of casein, paper and solvent must be so chosen as to give the best possible separation of the amino-acids on the paper. At present

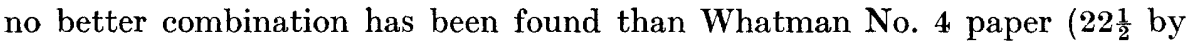
$18 \frac{1}{4}$ in.) with $n$-butanol + acetic acid mixture as solvent (Partridge, 1948). Adequate separation is obtained overnight (16 hr.), the solvent front usually passing off the paper in this time. For two-dimensional chromatography phenol is used as solvent for the second run at right angles to the first. Leucine cannot be separated from iso-leucine nor valine from methionine with this combination.

$n$-Butanol is redistilled before use and only the fraction distilling between 116 and $118^{\circ}$ is used. The $n$-butanol + acetic acid mixture is made by adding $30 \mathrm{ml}$. of glacial acetic acid (Analar) to $250 \mathrm{ml}$. of a 50/50 (v/v) mixture of $n$-butanol and water, in a graduated $250 \mathrm{ml}$. cylinder. After shaking, the mixture rapidly separates into two layers, the bottom one having a volume of $90 \mathrm{ml}$. Any marked variation from this volume indicates that something is wrong, usually that the butanol is impure or the acetic acid below strength. The amount of acetic acid added is fairly critical. The bottom aqueous layer (aqueous acetic acid saturated with butanol), is separated and placed in a beaker in the bottom of the tank. This should be changed for every run. The top, butanol, layer (butanol saturated with aqueous acetic acid), is used in the trough for running the chromatogram. It is not satisfactory to use this solvent again as ester formation takes place; only sufficient should be made for the number of chromatograms to be run at one time.

Any good-grade phenol is usually satisfactory, and a trace of colour does not seem to affect the separation of the amino-acids or to produce an unsatisfactory solvent front. It was found convenient to buy phenol in $500 \mathrm{~g}$. amounts and to liquefy this with $225 \mathrm{ml}$. water. On solution and shaking, an emulsion forms which often needs a day to clear; phenol saturated with water is the heavier phase; this is used in the trough. When a rapid separation of the emulsion is obtained, the material is likely to prove unsatisfactory. A beaker of water saturated with phenol is placed in the bottom of the tank; this need be changed only at long intervals.

When using phenol or $n$-butanol + acetic acid mixture a beaker of water is also placed in the tank as this aids the attainment of equilibrium conditions. Any phenol solution remaining in a trough after a run can be returned to a stock bottle and used again after resaturating with water. 'Collidine', a crude product consisting mainly of trimethylpyridines, has also been used. This material needs purification before use and because of its unpleasant penetrating odour, its greater sensitivity to changes of temperature than either $n$-butanol + 
acetic acid mixture or phenol, and the difficulty in eluting material from chromatograms run with it is for this reason not favoured in this laboratory.

Temperature. Because the solvents used are saturated with water at a definite temperature, any large temperature variations during a run may cause the appearance of a second phase consisting of water saturated with solvent, or the solvent may not remain saturated. In either case unsatisfactory chromatograms are produced. As already mentioned, 'collidine' is particularly sensitive to such changes; a rise of less than $1^{\circ}$ will cause cloudiness in a water-saturated 'collidine' solution. Phenol and the $n$-butanol + acetic acid mixture are very much less affected by temperature changes and will tolerate variations of $\pm 2^{\circ}$ about a mean without the chromatograms being seriously affected. Chromatograms employing these last two solvents can be run between 15 and $30^{\circ}$ without much effect on the separation obtainable, the higher temperatures giving faster running chromatograms. The problem of temperature control therefore resolves itself, with these two solvents, into holding tank temperatures steady to within $\pm 2^{\circ}$ of any temperature between 15 and $30^{\circ}$ for a period of about $16 \mathrm{hr}$. This was possible under all but the most exceptional climatic conditions by keeping the tanks in an ordinary room with all windows closed and heating the room electrically, the electric heaters being thermostatically controlled and of sufficient capacity to deal with the rapid drop in temperature sometimes experienced at night at certain times of the year. The tanks should stand well away from the windows.

Both phenol and $n$-butanol + acetic acid mixture run off the paper in $16 \mathrm{hr}$. Often this does not matter, but it may be necessary at times to study material moving very close to the solvent front. By adjusting the amount of solvent in the trough it is possible to regulate the distance the solvent front travels; $25 \mathrm{ml}$. is sufficient to allow the solvent front to remain on the paper when the chromatogram is run along the $22 \frac{1}{2}$ in. length of a sheet of No. 4 Whatman paper. The paper should, however, be removed from the tank as soon as possible after the front has become stationary; otherwise the spots lose their sharpness because of diffusion.

Preparation of paper. When comparisons have to be made of single-dimensional chromatograms of culture-filtrates run for various periods, some standardization of spot size and, therefore, of volume of material put on the paper at the starting line, is necessary. This may be done without difficulty by a very simple procedure. Chromatograms are normally run in the long direction of the paper. A base line is drawn at right angles to the $22 \frac{1}{2}$ in. edge. The distance of this line from the top of the paper is such that the spots will be clear of the glass supporting-rod and on the side away from the trough when the paper is in position. Positions for spots are marked along this line, starting 1 in. from the side and with $\frac{3}{4}$ in. between spots. At 0.2 in. from this line and parallel to it a second line is drawn. A short length of capillary tubing drawn to a fine point is filled by dipping its tip into the culture-filtrate to be examined. The tip of the capillary is then applied to the paper at one of the marked spots and removed when the circumference of the circle of liquid formed reaches the second line. With this technique, spots with a volume of about $0.004 \mathrm{ml}$. and 
of standard size can be placed on the paper. If a solution is considered to be too weak to give a satisfactory chromatogram, more spots are added at the same site, each spot being allowed to dry before each new addition.

With two-dimensional chromatograms a similar technique is employed but the spot size is decreased to a diameter of $0.1 \mathrm{in}$., since more satisfactory chromatograms are obtained with this smaller spot. No hard and fast rules can be made about the amount of material needed to obtain a chromatogram of satisfactory strength. As a rough guide, however, it was found that one spot of a culture medium containing about $8 \mu \mathrm{g}$. amino-nitrogen will give an excellent singledimensional picture with $n$-butanol-acetic acid mixture as solvent. Ninhydrin colours are weaker after two-dimensional running and the spots tend to be less sharply defined. Between 50 and $100 \mu \mathrm{g}$. amino-nitrogen were found necessary to give satisfactory two-dimensional pictures with the same culture filtrate.

Drying of papers. After a solvent-run papers are dried by hanging them in a steam-heated chamber. This was constructed from a bacteriological steamer approximately $3 \mathrm{ft}$. long, $2 \mathrm{ft}$. 6 in. wide and $2 \mathrm{ft}$. deep standing on one end so that the 'lid' now forms a vertical door ( $3 \mathrm{ft}$. high by $2 \mathrm{ft} .6 \mathrm{in}$. wide) hinged at one side. The chamber is heated internally by steam pipes and is lagged externally. The internal temperature attained is about $80^{\circ}$. The door is made fume-proof by a rubber gasket, and can be tightly closed by means of two bolts with butterfly nuts at top and bottom. A small centrifugal blower feeds air into the bottom of the chamber and the fumes pass through a $2 \mathrm{in}$. stainless steel pipe in the top of the chamber, led outside the building. With this apparatus a dozen papers can be dried with almost no escape of fumes into the room other than those arising when the papers are transferred from the tanks to the dryer.

After running the chromatogram the troughs with the papers are removed from the tank. The papers are suspended by 'bulldog' clips from the bottom edge in the hot chamber; to prevent tearing, a strip of dry filter-paper is used to strengthen this edge before applying the clips. Drying is then continued until the amino-acid and peptide spots examined under ultra-violet light show a reasonably strong fluorescence. At $70^{\circ}-80^{\circ}$ the drying usually takes from 2 to $3 \mathrm{hr}$. The examination under ultra-violet light, besides indicating when a paper is ready for spraying, may give other useful information; for instance, it is possible to detect flavins (Crammer, 1948; Woiwod \& Linggood, 1948), porphyrins (Rimington, 1948), and fluorescent bacterial pigments.

Colour development. A solution of ninhydrin in chloroform $(0 \cdot 1 \%, \mathrm{w} / \mathrm{v})$, with $\mathbf{0} \cdot \mathbf{1} \%$ 'collidine' added, is the most satisfactory reagent for spraying the papers. No other solvents so far tested produce such strong colours. The reason for this is not clear, but it is possible that chloroform modifies the complex reaction taking place between amino-acids and peptides and the ninhydrin. Dried sheets are removed to a fume-cupboard and sprayed, the operator wearing a respirator. The paper is returned to the heating chamber and the developed spots examined after about $15 \mathrm{~min}$., the heating being continued for another hour to ensure maximal development of colour. The small percentage of 'collidine' aids colour differentiation and is particularly useful in distinguishing glycine and 
serine when using the $n$-butanol + acetic acid mixture. Both these amino-acids have very similar $\boldsymbol{R}_{F}$ values in this solvent and produce very similar colours with ninhydrin in chloroform. In the presence of a trace of 'collidine', however, serine produces a blue coloration and glycine is dull red. It has thus been found possible to follow on single-dimensional chromatograms the disappearance of one or the other acid from a medium containing both.

The use of chloroform raises problems when large numbers of sheets have to be sprayed. Unless the spraying can be done in an efficient fume chamber and the operator works in a respirator, it is probably more satisfactory to use $n$-butanol as a solvent for the ninhydrin. The spray should not be too fine, as the amount of ninhydrin on the paper before heating has a marked effect on colour development, and subsequent respraying and heating will not intensify a chromatogram. A volume of $75 \mathrm{ml}$. is needed to spray adequately a sheet $22 \frac{1}{2}$ by $18 \frac{1}{4} \mathrm{in}$. This is achieved by two sprayings, a procedure advisable with chloroform as it dries so rapidly that portions of the chromatogram can be missed in the first spraying.

Recording of results. The most satisfactory records are photographs, a written note being made of those points which, because of shades of colour or for other reasons, will not be obvious on the print. Colour photography has proved disappointing and is not recommended.

\section{General observations}

The achievement of stable conditions of saturation in the tanks is of prime importance in obtaining satisfactory chromatograms. To this end it is most important not to leave tanks open longer than is necessary when removing troughs and papers. For the same reason it is not advisable to use any given tank for more than one solvent; it often takes a day or more to regain equilibrium after changing solvents. Unsatisfactory results may also be obtained when first using a new tank, but after once coming to equilibrium with a given solvent there is no further difficulty.

With the techniques described it is possible to run ten culture-filtrates and two controls of uninoculated medium in duplicate on one sheet, using the $18 \frac{1}{4}$ in. edge as starting line. As each tank holds three troughs and will if necessary hold four, up to 200 single-dimensional chromatograms can be run each night in one tank.

\section{REFERENCES}

Consden, R., Gordon, A. H. \& Martin, A. J. P. (1944). Qualitative analysis of proteins: a partition chromatographic method using paper. Biochem. J. 38, 224.

Crammer, J. L. (1948). Paper chromatography of flavine nucleotides. Nature, Lond., $161,349$.

LingGood, F. V. \& WoIwoD, A. J. (1948). The application of paper partition chromatography to the production of diphtheria toxin. Brit. J. exp. Path. 29, 283.

Partridge, S. M. (1948). Filter-paper partition chromatography of sugars. Biochem J. 42, 238. 
Proom, H. \& WoIwod, A. J. (1949a). Group relationships between bacteria shown by amino-acid metabolism. J. gen. Microbiol. 3, xiv.

Proom, H. \& Worwod, A. J. (1949b). The examination, by partition paper chromatography, of the nitrogen metabolism of bacteria. J. Gen. Microbiol. 3, 319.

Riming'Ton, C. (1948). Symposium on partition chromatography and its application to biochemical problems. Biochemical Society, October 1948.

Worwon, A. J. \& Proom, H. (1948). The examination of bacterial filtrates by paper chromatography. J. gen. Microbiol. 2, xxxi.

Worwod, A. J. \& LingGood, F. V. (1948). Production of riboflavin and allied substances during growth of C. diphtheriae. Nature, Lond., 162, 219.

(Received 26 November 1948) 